\section{Documentation of alcohol, cigarette and cannabis use by psychiatric and general practice trainees}

Alcohol misuse and the consumption of cigarettes and cannabis are associated with well-established impairments in physical and psychological health. Excessive consumption of these substances in Ireland now poses a major public health problem.

Per capita consumption of alcohol in Ireland increased by $41 \%$ between 1989 and 1999 , the highest such increase among EU countries, leaving Ireland with the second highest alcohol consumption levels of all EU countries. ${ }^{1}$ Tobacco smoke remains the leading preventable cause of death and disability in Ireland. ${ }^{2}$ While statistics on cannabis use are less clear, a recent UN study found Ireland to have the highest levels of cannabis use in the 23 countries of Western Europe. $^{3}$

Healthcare workers have an important role in recording levels of consumption, detecting abuse of these substances and promoting awareness of their adverse effects. Accurate recording of alcohol, cigarette and cannabis use at the time of admission to hospital is vital if problems in these areas are to be detected and managed during and after admission. Furthermore, screening and brief interventions have been shown to increase cigarette smoking cessation rates ${ }^{4}$ and have beneficial effects in cases of alcohol abuse. ${ }^{5}$

St Loman's Hospital was a 92-bed psychiatric hospital serving a catchment area population of 360,000 people in Dublin west. Some 79 beds were available for acute admissions with 13 beds reserved on a sub-acute/rehabilitation ward. Twelve non-consultant hospital doctors (NCHDs), nine of whom were psychiatric trainees and three of whom were general practice trainees performed admissions in the hospital within each six-month period.

We aimed to establish the level of recording of use of alcohol, cigarettes and cannabis in the admission notes of NCHDs working in St Loman's Hospital over a one-year period from September 1,1997.

Some 249 consecutive admission notes were examined retrospectively for a one-year period from September 1,1997. It was noted whether the admitting clinician was a psychiatric trainee, a general practice trainee or a consultant psychiatrist. Recording of alcohol, tobacco and cannabis use was classified as:

(0) Not recorded

(1) Descriptive record only eg. 'social drinker'

(2) Quantitative record, eg. '12 units per week.

The patient's age, sex, legal status (voluntary patient or detained under the Mental Treatment Act) and order of admission (first admission or readmission) were recorded. Results were analysed and $p$-values calculated for each category.

Of the 249 admissions, 186 were completed by psychiatric trainees, 58 by general practice trainees and three by consultant psychiatrists. Two admission notes were unsigned. Some 120 male and 129 female patients were admitted during the one-year period. Fifteen of the patients admitted were over the age of 65 years (eight male and seven female). Sixteen people were admitted as temporary patients under The Mental Treatment Act.

$P$-values were computed using the Sattxact 4 for Windows software for exact non-parametric inference. There was no statistical difference in the quality of recording between psychiatric and general practice trainees, between male and female patients, between patients aged under and over 65 and between voluntary and temporary patients.

People who had previously been admitted to St. Loman's Hospital were much less likely to have a history of tobacco use recorded than patients admitted for the first time $(p<$ 0.01 ). This is the first audit of its kind to be reported in an Irish setting. The low levels of recording of alcohol, cigarette and cannabis use are consistent with findings from other studies. ${ }^{6,7}$

Use of alcohol, cigarettes and cannabis now poses a major public health problem in Ireland. NCHDs have a central role in identifying and recording such problems, providing brief intervention and promoting healthy attitudes.

Medical education has been shown to lead to improvements in the detection and management of alcohol use by hospital interns. ${ }^{8}$ Medical education is also likely to lead to improvements in the detection and management of cigarette and cannabis use. We recommend comprehensive coverage of these issues in induction programmes for NCHDs taking up posts in psychiatry and other specialities.

*Henry O’Connell, MRCPsych, Research Fellow,

Mercer's Institute for Research on Ageing,

St. James's Hospital, Dublin 8, Ireland.

Majella Cahill, MRCPsych, Research Fellow,

Department of Psychiatry, Beaumont Hospital, Dublin 9, Ireland.

Denis Murphy, MRCPsych,

Consultant Psychiatrist,

Department of Psychiatry,

Adelaide and Meath Hospital incorporating The National Children's Hospital, Tallaght, Dublin 24, Ireland.

${ }^{*}$ Correspondence

References

1. Strategic Task Force on Alcohol-Interim Report. Department of Health, Ireland. 2002. 2. Office of Tobacco Control, Ireland. www.otc.ie

3. Global Illicit Trends, United Nations, 2002.

4. Bernstein SL, Becker BM. Preventive care in the emergency department: diagnosis and management of smoking and smoking-related illness in the emergency department: a systematic review. Acad Emerg Med 2002; 9(7): 720-9.

5. D'Onofrio G, Degutis LC. Preventive care in the emergency department: screening and brief intervention for alcohol problems in the emergency department: a systematic review. Acad Emerg Med 2002; 9(6): 627-38.

6 . Sheahan SL. Documentation of health risks and health promotion counselling by emergency department nurse practitioners and physicians. J Nurs Scholarship 2000; 32(3): 245-50.

7. Rowland N, Maynard AK, Kennedy PF, Wintersgill W, Stone WD. Teaching doctors to take alcohol histories: a limited success story. Med Educ 1988; 22(6):539-42.

8. Gaughwin M, Dodding J, White JM, Ryan P. Changes in alcohol history taking and management of alcohol dependence by interns at The Royal Adelaide Hospital. Med Educ 2000; 34(3):170-4. 